Revista Brasil. Bot., V.33, n.1, p.51-60, jan.-mar. 2010

\title{
Germinação de Pyrostegia venusta (Bignoniaceae), viabilidade de sementes e desenvolvimento pós-seminal
}

\author{
DAVI RODRIGO ROSSATTO ${ }^{1}$ e ROSANA MARTA KOLB ${ }^{1,2}$
}

(recebido: 18 de setembro de 2008; aceito: 11 de novembro de 2009)

\begin{abstract}
Germination of Pyrostegia venusta (Bignoniaceae), seed viability and post-seminal development). The effect of light and temperature on the germination of Pyrostegia venusta (Ker Gawl.) Miers was studied by isothermic incubations. The seeds of this species do not present photoblastism, and can germinate equally in the presence and absence of light. The minimum temperature for germination is between 10 and $15^{\circ} \mathrm{C}$ and the maximum between 35 and $40{ }^{\circ} \mathrm{C}$. The germination rate was higher at $35^{\circ} \mathrm{C}$, despite the fact that no statistically significant differences in the germinability were observed at 25 , 30 and $35^{\circ} \mathrm{C}$. At $25^{\circ} \mathrm{C}$, germination initiates at the $7^{\text {th }}$ day, while the development of the first pair of leaves occurs after 22 days of experiment. The seeds viability is better maintained when these are stored in glass containers at low temperatures. Germination in a wide range of temperature, and the absence of photoblastism allow the species to germinate in open areas and under the canopy, favoring its distribution in the different physiognomies of the Cerrado biome.
\end{abstract}

Key words - Cerrado, germination, Pyrostegia, seed storage, seed viability

RESUMO - (Germinação de Pyrostegia venusta (Bignoniaceae), viabilidade de sementes e desenvolvimento pós-seminal). O efeito da luz e da temperatura na germinação de Pyrostegia venusta (Ker Gawl.) Miers foi estudado por meio de incubações isotérmicas. As sementes da espécie não são fotoblásticas, podendo germinar igualmente na presença e na ausência de luz. A temperatura mínima para a germinação está entre 10 e $15^{\circ} \mathrm{C}$ e a máxima entre 35 e $40{ }^{\circ} \mathrm{C}$. As maiores taxas de germinação foram obtidas na temperatura de $35^{\circ} \mathrm{C}$, apesar de não haver diferenças estatisticamente significativas na germinabilidade em temperaturas de 25,30 e $35^{\circ} \mathrm{C}$. A $25^{\circ} \mathrm{C}$, a germinação tem início no $7^{\circ}$ dia, enquanto o desenvolvimento do primeiro par foliar ocorre a partir do $22^{\circ}$ dia de experimento. A viabilidade das sementes é mais bem mantida quando estas são armazenadas em recipientes de vidro a baixas temperaturas. A germinação em uma ampla faixa de temperatura, e a ausência de fotoblastismo permitem que a espécie germine em áreas abertas e sombreadas, propiciando sua distribuição pelas diferentes fisionomias do bioma Cerrado.

Palavras-chave - armazenamento, Cerrado, germinação, Pyrostegia, viabilidade de sementes

\section{Introdução}

A grande abrangência do bioma Cerrado garante a sua exposição a uma grande variedade de climas, solos e relevos que influem diretamente na ampla gama de formações vegetacionais e na diversidade de espécies (Oliveira-Filho \& Ratter 2002). O Cerrado brasileiro é caracterizado por sazonalidade pluviométrica, baixa disponibilidade nutricional no solo (Goodland \& Pollard 1973) e presença frequente de fogo (Coutinho 1978), sendo esses estresses marcantes na determinação das suas fisionomias e na composição florística (Ribeiro \& Walter 1998).

A sazonalidade pluviométrica no Cerrado é marcada por "verões" (outubro a abril) quentes e chuvosos e "invernos" (maio a setembro) frios e secos (Ratter et al. 1997). A alta sazonalidade do bioma influi diretamente

\footnotetext{
1. Universidade Estadual Paulista, Campus de Assis, Departamento de Ciências Biológicas, Faculdade de Ciências e Letras, Av. Dom Antonio, 2100, 19806-900 Assis, SP, Brasil.

2. Autor para correspondência: rosanakolb@hotmail.com
}

nos padrões reprodutivos e vegetativos das espécies nele presentes. Desta forma, as populações vegetais são altamente adaptadas aos fatores bióticos e abióticos do Cerrado (Mantovani \& Martins 1988, Franco 2002). Em relação ao processo germinativo, havendo disponibilidade de oxigênio e de água no solo, a temperatura e a luz são os fatores ambientais que mais influenciam na germinação das sementes (Felippe \& Silva 1984, Barbosa et al. 1999, Vieira et al. 2007).

Trabalhos vêm sendo realizados e têm confirmado a importância dos estudos de germinação com sementes de espécies do Cerrado, principalmente no que diz respeito à aquisição de conhecimentos nos processos de preservação das espécies e da regeneração de áreas degradadas, a partir das características ecofisiológicas da germinação (Felippe \& Silva 1984, Cesarino et al. 1998, Vieira et al. 2007). Além disso, as informações relacionadas à germinação das sementes têm contribuído para trabalhos em outras áreas do conhecimento, como estudos relacionados ao crescimento das espécies, conservação de germoplasma e tecnologia de sementes (Cesarino \& Zaidan 1998, Borghetti \& Ferreira 2004, Borghetti 2005). 
Pyrostegia venusta (Ker Gawl.) Miers, liana da família Bignoniaceae, conhecida pelo nome popular de cipó-de-são-joão, é comumente encontrada em formações savânicas e florestais do Cerrado brasileiro e está presente principalmente em bordas de Cerradão. Devido a sua presença em várias comunidades de Cerrado (Mantovani \& Martins 1988), de outras matas (Morelatto \& Leitão-Filho 1996, Udulutsch et al. 2004), e também em beira de estradas e outros ambientes perturbados (Lorenzi \& Souza 2001), a espécie torna-se componente importante das paisagens do bioma Cerrado. Essa espécie é utilizada como ornamental, não só no Brasil como em diversas partes do mundo, devido à produção abundante de flores de coloração laranja (Lorenzi \& Souza 2001). A espécie também tem utilização na medicina popular (Ferreira et al. 2000, Lorenzi \& Souza 2001, Di Stasi \& Hiruma-Lima 2003). Finalmente, as flores são ainda recursos importantes para beija-flores e psitacídeos, que se alimentam do néctar (Gobatto-Rodrigues \& Stort 1992).

O objetivo deste trabalho foi o de estudar os efeitos da luz e da temperatura na germinação da espécie, acompanhar o desenvolvimento pós-seminal (emergência do epicótilo e formação do primeiro par foliar) e testar diferentes formas de armazenamento das sementes, para responder as seguintes perguntas: a germinação das sementes de $P$. venusta é dependente da temperatura e da luminosidade? Qual o tempo decorrido desde a germinação e a formação das plântulas? Entre as condições de armazenamento: saco de papel ou vidro e/ou temperatura ambiente ou $10^{\circ} \mathrm{C}$, quais as condições com as melhores respostas? Os dados obtidos visam fornecer importantes informações da ecofisiologia da germinação das sementes e também contribuir com dados que subsidiem a propagação e consequente produção de mudas desta espécie, que apresenta valor ecológico e econômico.

\section{Material e métodos}

As sementes de Pyrostegia venusta, utilizadas neste estudo, foram coletadas em outubro de 2004, a partir de 15 indivíduos presentes na Estação Ecológica de Assis, Assis, SP $\left(22^{\circ} 33^{\prime} 65^{\prime} \mathrm{S}, 50^{\circ} 22^{\prime} 29^{\prime \prime} \mathrm{W}\right)$ e em áreas de Cerrado nos arredores da Faculdade de Ciências e Letras, Unesp, Assis-SP $\left(22^{\circ} 32^{\prime} 45^{\prime \prime} \mathrm{S}, 50^{\circ} 22^{\prime} 98^{\prime \prime} \mathrm{W}\right)$. Os tratamentos foram montados logo após a coleta e a homogeneização das sementes, consistindo cada um deles de cinco placas de Petri (14 cm de diâmetro), forradas com papel de filtro umedecido com água destilada, contendo 20 sementes cada. As incubações isotérmicas entre 10 e $40^{\circ} \mathrm{C}$, com intervalos de $5 \pm 1{ }^{\circ} \mathrm{C}$, foram realizadas em câmaras de germinação da Eletrolab (modelo 102 FC), em condições de luz branca (fotoperíodo de 12 horas) ou escuro contínuo. Nos tratamentos sob luz, as placas foram mantidas a irradiância média de $4126,34 \pm 127,37 \mu \mathrm{W} \mathrm{cm}{ }^{-2}$, fornecida por quatro lâmpadas fluorescentes do tipo luz do dia de $20 \mathrm{~W}$ cada. As medidas de irradiância foram feitas na altura das placas, com uma célula solar desenvolvida pela Heliodinâmica, SP, acoplada a um multímetro digital Topis, modelo MIC-2200A. Para estudar o efeito do escuro, as placas de Petri foram colocadas dentro de dois sacos de plástico preto para evitar a exposição à luz, e a observação da ocorrência da germinação foi feita sob luz verde de segurança (Amaral-Baroli \& Takaki 2001). O critério utilizado para germinação foi a protrusão da radícula (comprimento mínimo de $1 \mathrm{~mm}$ ) e o monitoramento dos experimentos foi encerrado 5 dias após a estabilização das porcentagens. Para análise do desenvolvimento pós-seminal foram utilizadas 10 repetições com 20 sementes em cada placa de Petri. O experimento foi mantido sob fotoperíodo de 12 horas e temperatura de $25 \pm 1{ }^{\circ} \mathrm{C}$. Foram consideradas as etapas de emergência do epicótilo e a formação do primeiro par foliar.

No estudo de viabilidade, as sementes foram armazenadas em sacos de papel e em vidro à temperatura ambiente (aproximadamente $\left.25^{\circ} \mathrm{C}\right) \mathrm{e}$ em geladeira $\left(10 \pm 1^{\circ} \mathrm{C}\right.$ ) por $3 \mathrm{e}$ 6 meses. Após esses períodos, a germinabilidade foi avaliada sob fotoperíodo de 12 horas, a $25 \pm 1{ }^{\circ} \mathrm{C}$ (temperatura comumente utilizada nos estudos de viabilidade de sementes pós-armazenamento). Foram utilizadas 10 repetições por tratamento contendo 10 sementes cada.

A porcentagem de germinação (germinabilidade), o tempo médio $(\bar{t})$, a frequência relativa $(f i)$ e o índice de sincronização da germinação (U) foram calculados de acordo com Labouriau (1983) para cada temperatura, na luz e no escuro. As equações utilizadas foram: $\bar{t}=\sum_{\mathrm{i}=1}^{\mathrm{k}} \mathrm{niti} / \sum_{\mathrm{i}=1}^{\mathrm{k}} \mathrm{ni}$, onde $\mathrm{ni}$ = número de sementes que germinaram no tempo ti (não o número acumulado, mas o número referido para a i-ésima observação), $\mathrm{ti}=$ tempo entre o início do experimento e a i-ésima observação, $k$ = último tempo de germinação das sementes; $f i=\mathrm{ni} / \sum_{\mathrm{i}=1}^{\mathrm{k}}$ ni, onde ni $=$ número de sementes que germinaram no tempo ti (não o número acumulado, mas o número referido para a i-ésima observação), $\mathrm{k}=$ último tempo de germinação das sementes; $\mathrm{U}=-\sum_{\mathrm{i}=1}^{\mathrm{k}} f i \log _{2} f i$, onde $f i=$ frequência relativa de germinação, $\mathrm{k}=$ último dia de observação.

No experimento de viabilidade de sementes foram calculados apenas a porcentagem de germinação e o tempo médio. A porcentagem de germinação foi transformada em arco seno $(x / 100)^{0,5}$ por motivos de normalização e para obter homogeneidade entre as variâncias. Os dados de tempo médio e de sincronia não foram transformados por apresentarem normalidade dos resíduos (Kolmogorov-Smirnov $P>0,50$ ) e homogeneidade entre as variâncias (teste de Cochran $P>0,40)$. Os dados referentes ao efeito da luz e da 
temperatura foram analisados pelo teste estatístico ANOVA dois fatores seguido de teste de Tukey a alfa $=5 \%$, enquanto que para o experimento de armazenamento foi utilizado teste ANOVA três fatores seguido de teste de Tukey a alfa $=5 \%$ (Zar 1999).

\section{Resultados e discussão}

Efeito da luz e da temperatura na germinação - As sementes de $P$. venusta não são fotoblásticas, pois a luz não foi fator significativo no processo germinativo, levados em consideração a porcentagem de germinação $(F=3,69, P=0,072)$, o tempo médio $(F=1,53$, $P=0,22)$ e o índice de sincronização $(F=0,60$, $P=0,47)$. Também não houve, para as variáveis estudadas, interação significativa entre temperatura e luminosidade $(F<2,00, P>0,05)$ confirmando que as sementes não apresentam fotoblastismo, em qualquer uma das temperaturas estudadas, apesar da sensibilidade das sementes à luz poder ser modificada devido a interações com a temperatura de incubação (Kendrick 1976). Desta forma, de acordo com a classificação proposta por Takaki (2001), a germinação das sementes de $P$. venusta deve ser controlada principalmente pelo fitocromo A, através de uma resposta de fluência muito baixa (VLFR), onde o fotoequilíbrio das formas inativas e ativas de fitocromo pré-existentes nas sementes é capaz de promover a resposta germinativa.

A ausência de fotoblastismo é comum para diversas espécies de árvores e arbustos do Bioma Cerrado (Felippe \& Silva 1984, Felippe 1990a, Godoy \& Felippe 1992, Barbosa et al. 1999). A ausência de influência da luz nas variáveis aqui estudadas pode estar relacionada com a germinação das sementes da espécie em diferentes áreas, desde bordas até o interior de florestas, pois segundo Seghese et al. (1992), sementes com alta capacidade de germinação tanto na presença como na ausência de luz são características de espécies tropicais que germinam tanto a pleno sol como sob o dossel de florestas. Muitos autores relatam que a espécie geralmente é encontrada em áreas abertas (Gobatto-Rodrigues 1988, Lorenzi \& Souza 2001, Di-Stasi \& Hiruma-Lima 2003). Entretanto, há relatos da presença da espécie no interior de matas densas e antigas (Hora \& Soares 2002, Udulutsch et al. 2004), corroborando com os resultados de que a espécie é capaz de germinar em diferentes condições de luminosidade. O mesmo foi encontrado por Socolowsky \& Takaki (2004) para Jacaranda mimosifolia D. Don., também da família Bignoniaceae.

Embora $P$. venusta possa germinar em áreas abertas e fechadas, faz-se necessário um estudo futuro sobre o requerimento de luminosidade para o estabelecimento de suas plântulas, que também pode estar influindo na distribuição da espécie nos diferentes habitats.

A temperatura influenciou significativamente na germinabilidade $(F=58,76, P=0,00)$, que foi mínima a $15^{\circ} \mathrm{C}$ e máxima (entre 88 e $95 \%$ ) nas temperaturas de 25,30 e $35^{\circ} \mathrm{C}$, não havendo diferença estatisticamente significativa entre essas temperaturas. Não houve germinação nas temperaturas de 10 e $40{ }^{\circ} \mathrm{C}$ (figura 1), apesar de algumas espécies do Cerrado apresentarem germinação nestas temperaturas (Fellipe \& Silva 1984). Outros estudos também encontraram altas porcentagens de germinação para as lianas: $93 \%$ para Ipomoea asarifolia (Ders.) Roem \& Schult. (Kill \& Ranga 2003); $100 \%$ para Jacquemontia multiflora (Choisy) Hallier f. (Kill \& Ranga 2000) e 85\% para Dioscorea composita Hemsley (Viana \& Felippe 1986).

Alguns estudos com espécies vegetais nativas têm mostrado que a velocidade de germinação depende das condições de incubação das sementes (Lima et al. 1997, Santos \& Cardoso 2001). O efeito da temperatura no tempo médio de germinação $(F=280,17, P=0,00)$ foi

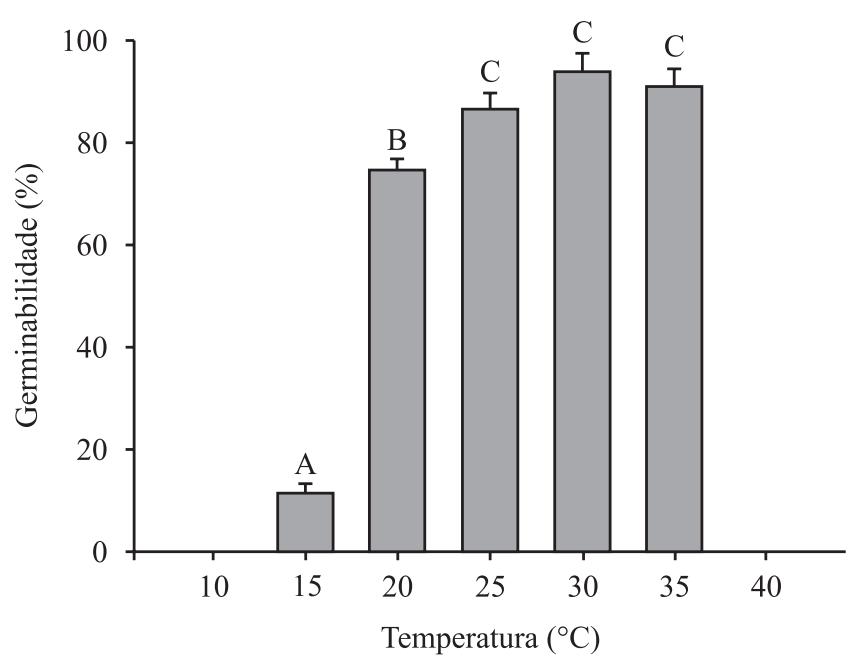

Figura 1. Porcentagem de germinação de sementes de Pyrostegia venusta em diferentes temperaturas. As barras representam a média \pm desvio padrão. Letras iguais indicam que não há diferença estatística entre os tratamentos de acordo com o teste de Tukey $(P<0,05)$. Por não haver diferença significativa entre os tratamentos na luz e no escuro, os dois tratamentos foram considerados em conjunto.

Figure 1. Germination percentage of Pyrostegia venusta seeds at different temperatures. Bars represent the mean \pm standard deviation. Same letters indicate that are not statistic difference among the treatments according Tukey's test $(P<0.05)$. As no significant differences were found between light treatments, both were grouped. 
significativo para $P$. venusta, sendo que quanto maior a temperatura (na faixa de 15 a $35^{\circ} \mathrm{C}$ ) menor o tempo médio para as sementes germinarem (figura 2). Logo, a temperatura ótima para essa espécie é a de $35^{\circ} \mathrm{C}$, que proporcionou alta germinabilidade e menor tempo médio de germinação. Segundo Borghetti \& Ferreira (2004) um menor tempo médio de germinação, ou seja, uma germinação rápida, é uma estratégia para que a espécie se estabeleça num determinado ambiente o mais rápido possível, aproveitando as condições ambientais favoráveis ao desenvolvimento de novos indivíduos. Em estudo fenológico, realizado na Estação Ecológica de Assis, nos anos de 2004 (mesmo local e ano de coleta das sementes de $P$. venusta) e 2005 , foi constatado que a espécie dispersa suas sementes entre os meses de julho e dezembro, com pico no mês de setembro, final da estação seca (D.R. Rossatto \& R.M. Kolb, dados não publicados), permitindo que a germinação ocorra assim que as chuvas se iniciem. Na primavera e no verão, desses mesmos anos, as temperaturas variaram entre 16 e $30^{\circ} \mathrm{C}$, sendo a temperatura média de $24^{\circ} \mathrm{C}$, aproximadamente (dados

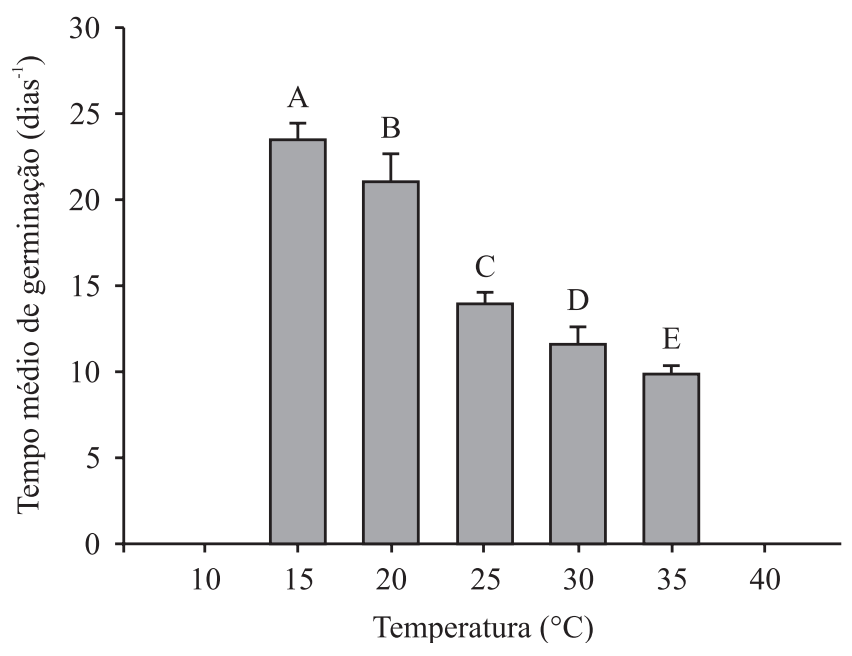

Figura 2. Tempo médio de germinação para sementes de Pyrostegia venusta em diferentes temperaturas. As barras representam a média \pm desvio padrão. Letras iguais indicam que não há diferença estatística entre os tratamentos de acordo com o teste de Tukey $(P<0,05)$. Por não haver diferença significativa entre os tratamentos na luz e no escuro, os dois tratamentos foram considerados em conjunto.

Figure 2. Mean germination time of Pyrostegia venusta seeds at different temperatures. Bars represent the mean \pm standard deviation. Same letters indicate that are not statistic difference among the treatments according Tukey's test $(P<0.05)$. As no significant differences were found between light treatments, both were grouped. obtidos no posto meteorológico da APTA regional médio Paranapanema em Assis, SP). Logo, a dispersão de sementes de $P$. venusta no final do inverno, pode propiciar uma germinação abundante devido ao aumento das temperaturas.

A frequência relativa foi mais heterogênea nas temperaturas mais baixas, sugerindo perda da sincronia no processo germinativo (Labouriau 1983). Porém, apresentou-se menos heterogênea nas temperaturas mais elevadas, tanto na presença quanto na ausência de luz (figura 3). Na temperatura ótima a frequência da germinação foi quase unimodal, estando em concordância com outros estudos (Ferraz-Grande \& Takaki 2001, Vieira et al. 2007).

O menor índice de sincronização foi observado na temperatura ótima (figura 4), mostrando haver uma maior sincronização da germinação nesta temperatura $(F=17,76, P=0,00)$. A estratégia de sincronizar o processo germinativo, assim como a de reduzir o tempo médio de germinação nas temperaturas ótimas, está relacionada com a rápida colonização do ambiente. Por outro lado, o atraso (figura 2) e a menor sincronia da germinação nas temperaturas mais baixas (figura 4), podem aumentar as chances de que pelo menos algumas sementes possam germinar em condições ambientais favoráveis ao estabelecimento das plântulas (Borghetti \& Ferreira 2004).

A temperatura, portanto, foi um fator importante influenciando todas as variáveis estudadas, modificando germinabilidade, tempo médio e sincronia da germinação das sementes. Outros trabalhos já vêm demonstrando o papel importante que a temperatura possui na germinação de espécies do Cerrado (Felippe \& Silva 1984, Borghetti \& Ferreira 2004, Vieira et al. 2007). O Cerrado possui uma grande abrangência geográfica e por isso está submetido a uma grande variação de climas, resultando numa alta variação de temperaturas (Gottsberger \& Silberbauer-Gottsberger 2006). Assim, a germinação em ampla faixa de temperatura (entre 15 e $35{ }^{\circ} \mathrm{C}$ ) pode propiciar e explicar, ao menos em parte, a ampla distribuição de $P$. venusta nas diferentes regiões de Cerrado, bem como em outras áreas brasileiras.

Germinação e desenvolvimento pós-seminal - As sementes de $P$. venusta iniciaram sua germinação entre o $7^{\circ}$ e o $8^{\circ}$ dias após o início dos experimentos, sendo que a maioria delas germinou entre o $10^{\circ}$ e o $21^{\circ}$ dias de experimento. A espécie levou cerca de 30 dias para alcançar a máxima porcentagem de germinação, tanto em condições de luz quanto em sua ausência, a $25^{\circ} \mathrm{C}$ (figura 5). A emergência do epicótilo foi observada na 

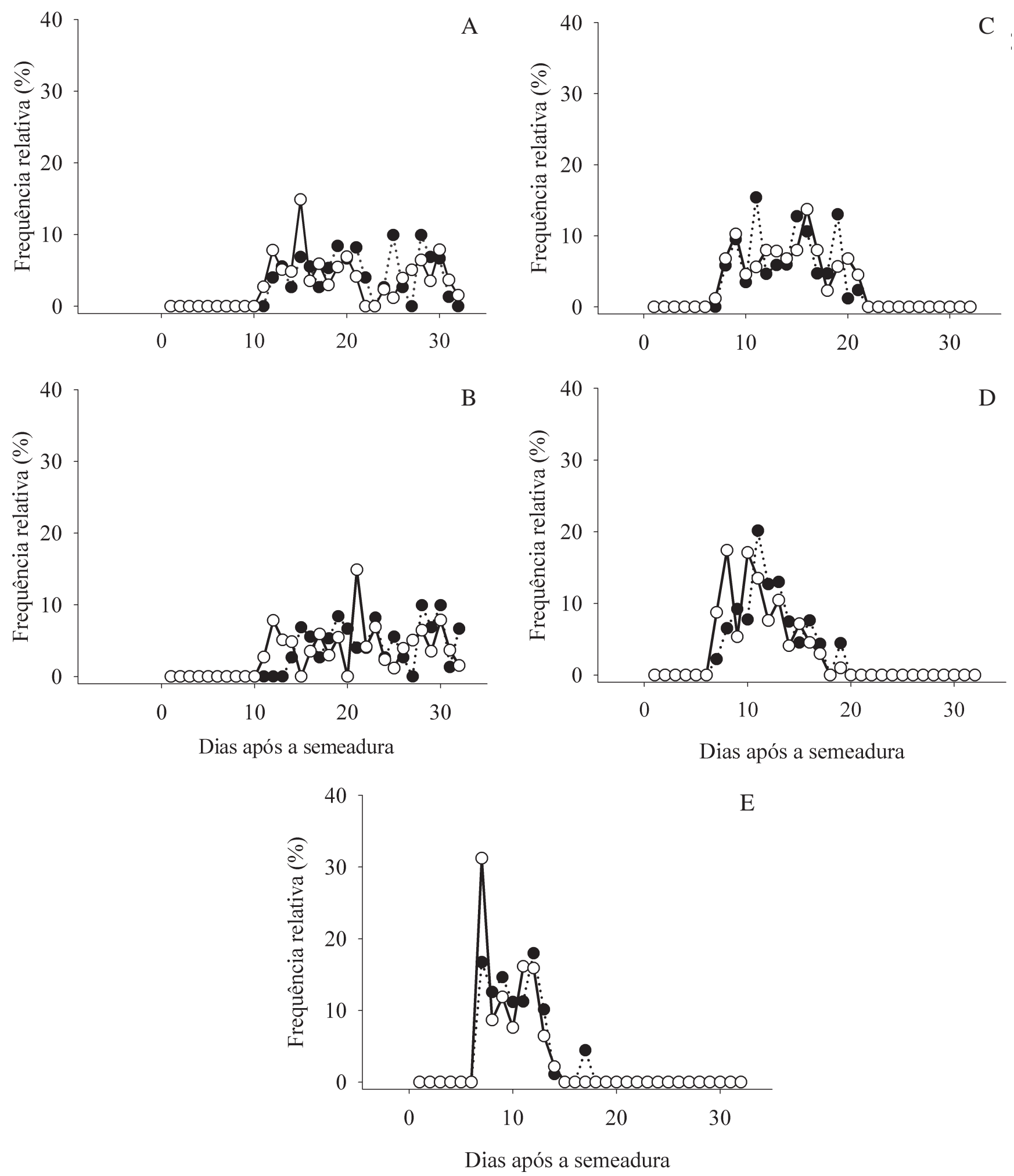

Figura 3. Frequência relativa da germinação de sementes de Pyrostegia venusta na luz $(O)$ e no escuro $(\bullet)$ em diferentes temperaturas. A. $15^{\circ} \mathrm{C}$. B. $20^{\circ} \mathrm{C}$. C. $25^{\circ} \mathrm{C}$. D. $30^{\circ} \mathrm{C}$. E. $35^{\circ} \mathrm{C}$.

Figure 3. Germination relative frequency of Pyrostegia venusta seeds under light $(\mathrm{O})$ and darkness $(\bullet)$ at different temperatures. A. $15^{\circ} \mathrm{C}$. B. $20^{\circ} \mathrm{C}$. C. $25^{\circ} \mathrm{C}$. D. $30^{\circ} \mathrm{C}$. E. $35^{\circ} \mathrm{C}$. 


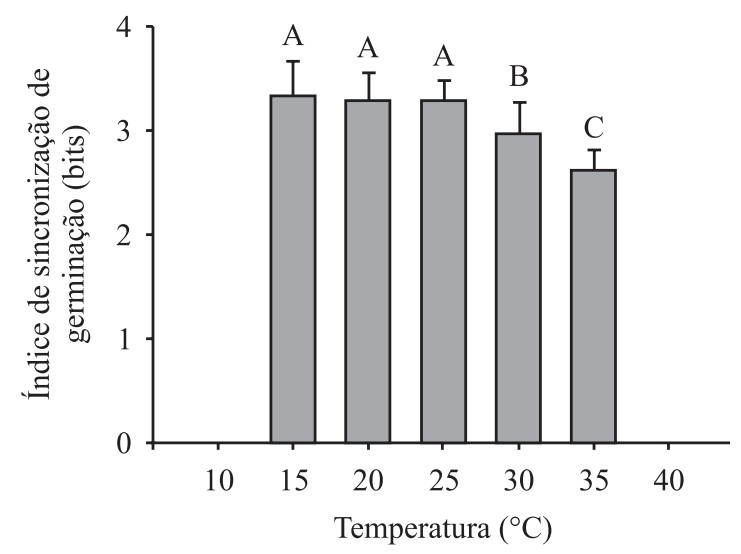

Figura 4. Índice de sincronização de germinação (U) das sementes de Pyrostegia venusta em diferentes temperaturas. As barras representam a média \pm desvio padrão. Letras iguais indicam que não há diferença estatística entre os tratamentos de acordo com o teste de Tukey $(P<0,05)$. Por não haver diferença significativa entre os tratamentos na luz e no escuro, os dois tratamentos foram considerados em conjunto

Figure 4. Germination synchronization index (U) of Pyrostegia venusta seeds at different temperatures. Bars represent the mean \pm standard deviation. Same letters indicate that are not statistic difference among the treatments according Tukey's test $(P<0.05)$. As no significant differences were found between light treatments, both were grouped luz no $18^{\circ}$ dia do experimento. Das 200 sementes que foram postas para germinar, $145(72,5 \%)$ emitiram o epicótilo na luz (figura 5).

O surgimento das folhas teve início a partir do $22^{\circ}$ dia do experimento. O número de plântulas que desenvolveram o primeiro par foliar em condições de luz foi de 113 , representando $56,5 \%$ do total das sementes postas para germinar e $91 \%$ das plântulas que apresentaram emergência do epicótilo (figura 5). A $25^{\circ} \mathrm{C}$, as sementes de Jacaranda caroba (Vell.) A. DC., outra bignoniácea do Cerrado, apresentaram comportamento germinativo semelhante ao de $P$. venusta, ou seja, iniciaram sua germinação no 7 o dia de experimento, sendo que a maioria das sementes já havia germinado após 30 dias. O surgimento das folhas teve início no $16^{\circ}$ dia de experimento e, das sementes que foram postas para germinar $74 \%$ formaram plântulas (A.P. Ansanelo \& R.M. Kolb, dados não publicados).

Os períodos para detecção da germinação das espécies do Cerrado são variáveis: Vochysia tucanorum Mart., a $25^{\circ} \mathrm{C}$, inicia sua germinação após 7 dias, levando em torno de 15 dias para atingir uma alta germinabilidade (Barbosa et al. 1999). Bidens gardneri Baker inicia sua germinação com 4 dias, apresentando maior germinabilidade após 20 dias (Felippe 1990b).

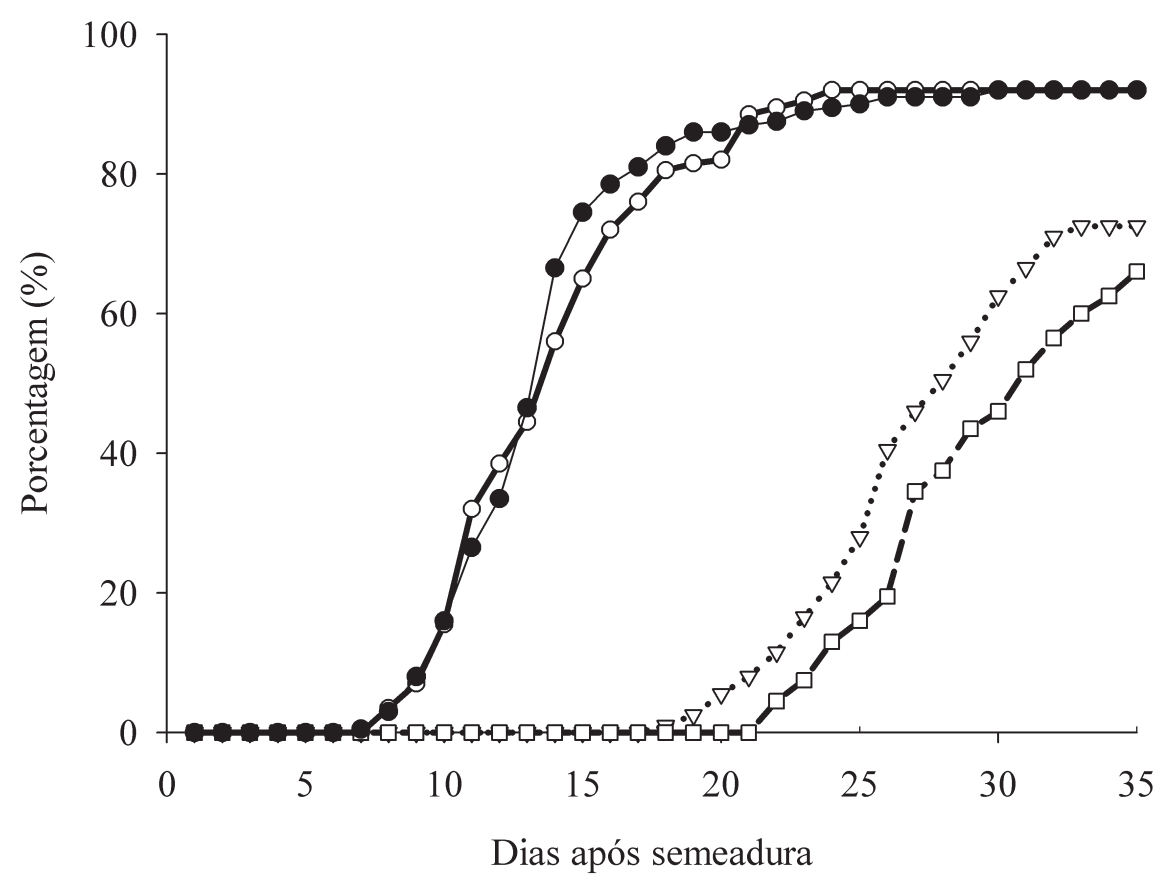

Figura 5. Germinação e desenvolvimento pós-seminal de Pyrostegia venusta a $25^{\circ} \mathrm{C}$. ( $\bullet$ ) germinação das sementes na ausência de luz; (O) germinação das sementes na presença de luz; $(\nabla)$ emergência do epicótilo na luz; $(\square)$ formação de folhas.

Figure 5. Germination and post-seminal development of Pyrostegia venusta at $25^{\circ} \mathrm{C}$. ( $\bullet$ ) seed germination in darkness; (O) seed germination under light; $(\nabla)$ epicothyl emergency in light; $(\square)$ leaf formation. 
Qualea grandiflora Mart. inicia sua germinação com 4 dias, atingindo a maior porcentagem no $10^{\circ}$ dia (Felippe 1990a). Stylosanthes macrocephala M.B. Ferreira \& Sousa Costa inicia sua germinação logo no primeiro dia de experimento, apresentando o máximo de germinação no $12^{\circ}$ dia (Silva \& Felippe 1986). Entretanto, em geral, as espécies iniciam sua germinação logo após ocorrer disponibilidade de água, germinando num curto período de tempo, assim como observado para as sementes de $P$. venusta. Quanto mais sementes germinarem em menor tempo, maior a probabilidade das plântulas se estabelecerem e da população aumentar, tornando-se bem distribuída e abundante, fato comum para muitas espécies de Cerrado (Felippe 2002).

Viabilidade das sementes armazenadas - Através da análise estatística ANOVA verificou-se que houve interação entre os três fatores analisados (tipo de embalagem, tempo e temperatura de armazenamento) na viabilidade das sementes de $P$. venusta $(F=10,89$, $P=0,00)$. Após 3 meses de armazenamento, houve manutenção da germinabilidade em relação ao experimento inicial (controle), apenas nos tratamentos mantidos a $10^{\circ} \mathrm{C}$. Houve queda significativa da porcentagem de germinação, quando comparada aos valores iniciais, para todos os tratamentos armazenados por 6 meses. No entanto, o melhor resultado foi obtido para sementes armazenadas em vidro a $10^{\circ} \mathrm{C}$, seguido daquelas armazenadas em embalagem de papel, também a $10{ }^{\circ} \mathrm{C}$ (tabela 1). Logo, para melhor manter a viabilidade das sementes de $P$. venusta, deve-se armazená-las em temperaturas mais baixas. Quanto ao tipo de embalagem, pode-se utilizar vidro ou papel para períodos menores de armazenamento (3 meses), desde que mantidos a baixas temperaturas. Para períodos maiores de armazenamento (6 meses), as sementes devem ser armazenadas somente em embalagem de vidro (tabela 1 ).
Gobatto-Rodrigues (1988) relatou que as sementes de $P$. venusta perderam a viabilidade após 5 meses quando armazenadas em sacos de papel a $25^{\circ} \mathrm{C}$. O resultado obtido no presente trabalho foi semelhante, no entanto, foi encontrada ainda uma porcentagem de germinação de $25 \%$ após 6 meses de armazenamento, nas mesmas condições citadas. Quando as sementes foram armazenadas em papel e a $10^{\circ} \mathrm{C}$, a viabilidade foi de $61 \%$ e, quando armazenadas em vidro, nesta mesma temperatura, a viabilidade foi de $67 \%$ (tabela 1 ).

$\mathrm{O}$ armazenamento em baixas temperaturas tem-se mostrado muito eficaz na manutenção da germinabilidade das sementes (Figliolia 1988, Figliolia et al. 2000), por manter um teor de água estável nos tecidos das sementes (Figliolia et al. 2000). A embalagem de vidro, por ser impermeável, pode ter auxiliado nessa função, principalmente nas sementes de $P$. venusta armazenadas por 6 meses. Outro possível fato favorável da refrigeração sobre o armazenamento seria a redução das reações metabólicas no embrião, conservando assim o vigor das sementes (Scalon et al. 2006).

Para manutenção do tempo médio de germinação das sementes de $P$. venusta, armazenadas por 3 meses, pode-se utilizar qualquer uma das embalagens e das temperaturas testadas (tabela 2). Não houve diferença estatisticamente significativa entre os resultados obtidos para os tratamentos com sementes armazenadas por 6 meses, porém o tempo médio de germinação aumentou significativamente em relação aos valores iniciais (tabela 2). Logo, no período mais longo de armazenamento, nenhuma das embalagens e das temperaturas testadas foi eficiente em manter o tempo médio de germinação. Cabral et al. (2003) estudando a viabilidade de sementes de Tabebuia aurea (Manso) Benth. \& Hook. F. ex. S. Moore, da família Bignoniaceae, armazenadas em câmara fria $\left(15^{\circ} \mathrm{C}\right)$ por curto período (3 meses),

Tabela 1. Porcentagem de germinação de sementes de Pyrostegia venusta após 3 e 6 meses de armazenamento.

Table 1. Germination percentage of seeds of Pyrostegia venusta stored for 3 and 6 months.

\begin{tabular}{|c|c|c|c|c|c|}
\hline \multirow{2}{*}{$\begin{array}{c}\text { Tempo } \\
\text { (meses) }\end{array}$} & \multicolumn{5}{|c|}{ Tratamentos } \\
\hline & $\begin{array}{l}\text { Papel } \\
25^{\circ} \mathrm{C}\end{array}$ & $\begin{array}{l}\text { Vidro } \\
25^{\circ} \mathrm{C}\end{array}$ & $\begin{array}{l}\text { Papel } \\
10^{\circ} \mathrm{C}\end{array}$ & $\begin{array}{l}\text { Vidro } \\
10^{\circ} \mathrm{C}\end{array}$ & Inicial \\
\hline 3 & $54 \mathrm{~d}$ & $60 \mathrm{c}$ & $77 \mathrm{a}$ & $78 \mathrm{a}$ & $90 \mathrm{a}$ \\
\hline 6 & $25 \mathrm{e}$ & $30 \mathrm{e}$ & $61 \mathrm{c}$ & $67 \mathrm{~b}$ & $90 \mathrm{a}$ \\
\hline
\end{tabular}

Valores seguidos da mesma letra não diferem estatisticamente pelo teste de Tukey $(P<0,05)$. Values followed by the same letter are not statistically different by Tukey's test $(P<0.05)$. 
Tabela 2. Tempo médio (dias) da germinação das sementes de Pyrostegia venusta após 3 e 6 meses de armazenamento.

Table 2. Mean germination time (days) of seeds of Pyrostegia venusta stored for 3 and 6 months.

\begin{tabular}{|c|c|c|c|c|c|}
\hline \multirow{2}{*}{$\begin{array}{c}\text { Tempo } \\
\text { (meses) }\end{array}$} & \multicolumn{5}{|c|}{ Tratamentos } \\
\hline & $\begin{array}{l}\text { Papel } \\
25^{\circ} \mathrm{C}\end{array}$ & $\begin{array}{l}\text { Vidro } \\
25^{\circ} \mathrm{C}\end{array}$ & $\begin{array}{l}\text { Papel } \\
10{ }^{\circ} \mathrm{C}\end{array}$ & $\begin{array}{l}\text { Vidro } \\
10{ }^{\circ} \mathrm{C}\end{array}$ & Inicial \\
\hline 3 & $10,40 \mathrm{a}$ & $10,29 \mathrm{a}$ & $12,01 \mathrm{a}$ & $11,22 \mathrm{a}$ & $11,82 \mathrm{a}$ \\
\hline 6 & $17,59 \mathrm{~b}$ & $19,44 \mathrm{~b}$ & $16,93 \mathrm{~b}$ & $16,94 \mathrm{~b}$ & $11,82 \mathrm{a}$ \\
\hline
\end{tabular}

Valores seguidos da mesma letra não diferem estatisticamente pelo teste de Tukey $(P<0,05)$. Values followed by the same letter are not statistically different by Tukey's test $(P<0.05)$.

encontraram resultados semelhantes aos de $P$. venusta, ou seja, manutenção da germinabilidade e da velocidade média da germinação independente da embalagem utilizada.

Os estudos têm mostrado que a longevidade de sementes de Cerrado, quando armazenadas em condições de laboratório, é bastante variável, podendo ser maior do que a observada para as sementes de $P$. venusta. Cesarino et al. (1998) mostraram que as sementes de Diplusodon virgatus Pohl (Lythraceae) mantêm sua viabilidade praticamente inalterada por quase 12 meses quando armazenadas em baixas temperaturas, principalmente devido à presença de um tegumento duro e impermeável. Para Vernonia cognata L. (Asteraceae) (Cesarino \& Zaidan 1998), o armazenamento a baixas temperaturas foi eficiente na manutenção da porcentagem de germinação das suas sementes por um período de 8 meses. Quando armazenadas a $25{ }^{\circ} \mathrm{C}$ as sementes de $V$. cognata perdiam rapidamente a viabilidade após 2 meses de armazenamento.

Sassaki et al. (1999a, b) mostraram que sementes de espécies de Cerrado podem ficar armazenadas no solo por 10 a 12 meses sem germinar, formando assim bancos persistentes de sementes. Os autores atribuem a um tegumento duro e impermeável a responsabilidade pela manutenção destas sementes nos bancos do solo. É provável que $P$. venusta não seja capaz de formar tais bancos, tendo em vista que suas sementes não apresentam tegumento impermeável (Gabrielli \& Castro 1995), que sua germinação ocorre rapidamente após contato com água, e devido à perda de viabilidade, relativamente rápida, após armazenamento das sementes em temperatura ambiente $\left(25^{\circ} \mathrm{C}\right)$.

Diante dos resultados aqui obtidos, foi observado que a luz não influencia o processo germinativo de $P$. venusta, entretanto as diferentes temperaturas possuem uma grande influência na germinação. As sementes são viáveis por pelo menos 6 meses, quando armazenadas em baixas temperaturas e em frascos de vidro fechados. Ainda, a formação de plântulas em condições controladas é rápida, ocorrendo aproximadamente 30 dias após a semeadura, propiciando o cultivo da espécie.

Agradecimentos - À Fundação de Amparo à Pesquisa do Estado de São Paulo (Fapesp), pela bolsa concedida ao primeiro autor, Processo 04/12585-4 e ao Dr. Aldo L. Klein (in memorian), pela orientação nos estágios iniciais deste trabalho.

\section{Referências bibliográficas}

AMARAL-BAROLI, A. \& TAKAKI, M. 2001. Phytochrome controls achene germination in Bidens pilosa L. (Asteraceae) by very low fluence response. Brazilian Archives of Biology and Technology 44:121-124.

BARBOSA, A.R., YAMAMOTO, K. \& VÁLIO, I.F.M. 1999. Effect of light and temperature on germination and early growth of Vochysia tucanorum Mart., Vochysiaceae, in cerrado and forest soil under different radiation levels. Revista Brasileira de Botânica 22:275-280.

BORGHETTI, F. 2005. Temperaturas extremas e a germinação de sementes. In Estresses ambientais, danos e benefícios em plantas (R.J.M.C. Nogueira, E.L. Araújo, L.G. Willadino \& U.M.T. Cavalcante, eds.). MXM Gráfica e Editora, Recife, p.207-218.

BORGHETTI, F. \& FERREIRA, A.G. 2004. Interpretação de resultados de germinação. In Germinação: do básico ao aplicado (A.G. Ferreira \& F. Borghetti, orgs.). Artmed, Porto Alegre, p.209-222.

CABRAL, E.L., BARBOSA, D.C.A. \& SIMABUKURO, E.A. 2003. Armazenamento e germinação de sementes de Tabebuia aurea (Manso) Benth. \& Hook. f. ex. S. Moore. Acta Botanica Brasilica 17:609-617.

CESARINO, F.C. \& ZAIDAN, L.B.P. 1998. Vernonia cognata Less. (Asteraceae): armazenamento e viabilidade de aquênios. Hoehnea 25:59-70.

CESARINO, F.C., ARAUJO, J.E. \& ZAIDAN, L.B.P. 1998. Germinação de sementes e crescimento de plantas de Diplusodon virgatus Pohl, Lythraceae. Acta Botanica Brasilica 12:349-356. 
COUTINHO, L.M. 1978. O conceito de cerrado. Revista Brasileira de Botânica 1:17-23.

DI STASI, L.C. \& HIRUMA-LIMA, C.A. 2003. Plantas medicinais na Amazônia e mata atlântica. Editora da Universidade Estadual Paulista, São Paulo.

FELIPPE, G.M. 1990a. Qualea grandiflora: the seed and its germination. Revista Brasileira de Botânica 13:33-37.

FELIPPE, G.M. 1990b. Germinação de Bidens gardneri, uma planta anual dos cerrados. Hoehnea 17:7-11.

FELIPPE, G.M. 2002. Desenvolvimento de plantas do cerrado: uma experiência pessoal. In Eugen Warming e o cerrado brasileiro: Um século depois (A.L. Klein, org.). Editora Unesp, São Paulo, p.107-120.

FELIPPE, G.M. \& SILVA, J.C.S. 1984. Estudos de germinação em espécies do cerrado. Revista Brasileira de Botânica 7:157-163.

FERRAZ-GRANDE， F.G.A. \& TAKAKI， M. 2001. Temperature dependent seed germination of Dalbergia nigra Allem. (Leguminosae). Brazilian Archives of Biology and Technology 44:401-404.

FERREIRA, D.T., ALVARES, P.S.M., HOUGHTON, P.J. \& BRAZ-FILHO, R. 2000. Constituintes químicos das raízes de Pyrostegia venusta e considerações sobre sua importância medicinal. Química Nova 23:42-46.

FIGLIOLIA, M.B. 1988. Conservação de sementes de essências florestais. Boletim Técnico do Instituto Florestal 42:1-18.

FIGLIOLIA, M.B., SILVA, A., AGUIAR, I.B. \& PERECIN, D. 2000. Conservação de sementes de Cariana estrellensis Kuntze em diferentes condições de acondicionamento e armazenamento. Revista Árvore 24:361-368.

FRANCO, A.C. 2002. Ecophysiology of woody plants. In The cerrados of Brazil (P.S. Oliveira \& R.J. Marquis, eds.). Columbia University Press, New York, p.178-197.

GABRIELLI, A.C. \& CASTRO, M.M. 1995. Anatomia da semente madura de Pyrostegia venusta (Ker.) Miers Bignoniaceae. Revista Brasileira de Botânica 18: 227-234.

GOBATTO-RODRIGUES, A.A. 1988. Biologia da reprodução de Pyrostegia venusta (Ker-Gawl) Miers (Bignoniaceae). Tese de mestrado, Universidade Estadual Paulista, Rio Claro.

GOBATTO-RODRIGUES, A.A. \& STORT, M.N.S. 1992. Biologia floral e reprodução de Pyrostegia venusta (Ker-Gawl) Miers (Bignoniaceae). Revista Brasileira de Botânica 15:37-41.

GODOY, S.M.A. \& FELIPPE, G.M. 1992. Qualea cordata: a semente e sua germinação. Revista Brasileira de Botânica 15:17-22.

GOODLAND, R. \& POLLARD, R. 1973. The Brazilian cerrado vegetation: a fertility gradient. Journal of Ecology 61:219-224.

GOTTSBERGER, G. \& SILBERBAUER-GOTTSBERGER, I. 2006. Life in the cerrado: a South American tropical seasonal ecosystem, v.1, Reta Verlag, Ulm.
HORA, R.C. \& SOARES, J.J. 2002. Estrutura fitossociológica da comunidade de lianas em uma floresta estacional semidecidual na Fazenda Canchim, São Carlos, SP. Revista Brasileira de Botânica 25:323-329.

KENDRICK, R.E. 1976. Photocontrol of seed germination. Science Progress 63:347-367.

KILL, L.H.P \& RANGA, N.T. 2000. Biologia floral e sistema de reprodução de Jacquemontia multiflora (Choisy) Hallier f. (Convolvulaceae). Revista Brasileira de Botânica 23:37-43.

KILL, L.H.P. \& RANGA, N.T. 2003. Ecologia da polinização de Ipomoea asarifolia (Ders.) Roem \& Schult (Convolvulaceae) na região semi-árida de Pernambuco. Acta Botanica Brasilica 17:355-362

LABOURIAU, L.G. 1983. A germinação das sementes. Série de Biologia. Monografia 24. Secretaria Geral da Organização dos Estados Americanos - OEA, Washington.

LORENZI, H. \& SOUZA, H.M. 2001. Plantas ornamentais no Brasil. Instituto Plantarum, Nova Odessa.

LIMA, C.M.R., BORGHETTI, F. \& SOUSA, M.V. 1997. Temperature and germination of the leguminosae Enterolobium contortisiliquum. Revista Brasileira de Fisiologia Vegetal 9:97-102.

MANTOVANI, W. \& MARTINS, F.R. 1988. Variações fenológicas das espécies do cerrado da Reserva Biológica de Moji-Guaçu, Estado de São Paulo. Revista Brasileira de Botânica 11:101-112.

MORELLATO, L.P.C. \& LEITÃO-FILHO, H.F. 1996. Reproductive phenology of climbers in a Southeastern Brazilian Forest. Biotropica 28:180-191.

OLIVEIRA-FILHO, A.T. \& RATTER, J.A. 2002. Vegetation physiognomies and woody flora of the Cerrado Biome. In The cerrados of Brazil (P.S Oliveira \& R.J. Marquis, eds.). Columbia University Press, New York, p.121-140.

RATTER, J.A., RIBEIRO, J.F. \& BRIDGEWATER, S. 1997. The Brazilian cerrado vegetation and threats to its biodiversity. Annals of Botany 80:223-230.

RIBEIRO, J.F. \& WALTER, B.M.T. 1998. Fitofisionomias do Bioma Cerrado. In Cerrado: ambiente e flora (S.M. Sano \& S.P. Almeida, eds.). Embrapa/CPAC, Brasília, p.89-166.

SANTOS, D.L. \& CARDOSO, V.J.M. 2001. Thermalbiological aspects on seed germination of Cucumis anguria L.: influence of the seed coat. Revista Brasileira de Botânica 24:435-440.

SASSAKI, R.M., RONDON, J.N., ZAIDAN, L.B.P. \& FELIPPE, G.M. 1999a. Germination of seed from herbaceous plants artificially stored in cerrado soil. Revista Brasileira de Biologia 59:271-279.

SASSAKI, R.M., RONDON, J.N., ZAIDAN, L.B.P. \& FELIPPE, G.M. 1999b. Longevity of seed from legume tree species artificially stored in cerrado and forest soils. Hoehnea 26:29-45. 
SCALON, S.P.Q., MUSSURY, R.M., SCALON FILHO, H., FRANCELINO, C.S.F., FLORENCIO, D.K.A. 2006. Armazenamento e tratamentos pré-germinativos em sementes de Jacarandá (Jacaranda cuspidifolia Mart.). Revista Árvore 30:179-185.

SEGHESE, F., ISSHIKI, K. \& VITTI, A.P. 1992. Ecofisiologia da germinação de espécies arbóreas. Série Técnica IPEF 8:9-11.

SILVA, J.C.S. \& FELIPPE, G.M. 1986. Germination of Stylosanthes macrocephala. Revista Brasileira de Botânica 9:263-268.

SOCOLOWSKI, F. \& TAKAKI, M. 2004. Germination of Jacaranda mimosifolia (D. Don - Bignoniaceae) seeds: effects of light, temperature and water stress. Brazilian Archives of Biology and Technology 47: 785-792.
TAKAKI, M. 2001. New proposal of classification of seed based on forms of phytochrome instead of photoblastism. Revista Brasileira de Fisiologia Vegetal 13:103-107.

UDULUTSCH, R.G., ASSIS, M.A. \& PICCHI, D.G. 2004. Florística de trepadeiras numa floresta estacional semidecídua, Rio Claro - Araras, Estado de São Paulo, Brasil. Revista Brasileira de Botânica 27:125-134.

VIANA, A.M. \& FELIPPE, G.M. 1986. Efeitos de luz e da temperatura na germinação de Dioscorea composita. Revista Brasileira de Botânica 9:109-116.

VIEIRA, D.C.M, SOCOLOWSKI, F. \& TAKAKI, M. 2007. Germinação de sementes de Dyckia tuberosa (Vell.) Beer (Bromeliaceae) sob diferentes temperaturas em luz e escuro. Revista Brasileira de Botânica 30:183-188.

ZAR, J.H. 1999. Biostatistical analysis. Prentice-Hall, New Jersey. 